\title{
Diseño y aplicación de una unidad didáctica para la enseñanza aprendizaje del concepto de disoluciones, apoyada en un simulador de cultivos hidropónicos.
}

\begin{abstract}
Design and implementation of a didactic unit for the teaching learning of the concept dissolutions, supported in a hydroponic cultivation simulator.
\end{abstract}

Claudia Patricia Neusa, V. ${ }^{1}$, Angie Torres, $0 .{ }^{1}$ y Sandra Isabel Enciso, G. ${ }^{2}$

${ }^{1}$ Licenciadas en Química UDFJC, ${ }^{2}$ Directora del Proyecto y Docente UDFJC. Grupo de investigación GREECE, Proyecto Curricular de Licenciatura en Química. Universidad Distrital Francisco José de Caldas. Colegio Oficial Distrital Los Comuneros Oswaldo Guayasamín. E-mail patricia.neusa@gmail.com ${ }^{(1)}$ angieflacaquimika@yahoo.es ${ }^{(1)}$ sandra.enciso@gmail.com $^{(2)}$

\section{Resumen}

Esta investigación es importante por el interés que causa la utilización de nuevas tecnologías de la información y la comunicación (NIIC's) para la enseñanza de la química en las instituciones educativas, esto con el fin de dar a conocer que la tecnología en materia de informática, al trabajar mancomunadamente con la investigación educativa, pueden producir en conjunto material de apoyo para facilitar el proceso de enseñanza aprendizaje de los estudiantes en el aula.

Palabras clave

Unidad didáctica, resolución de problemas, NIIC's, software educativo, investigación acción participativa.

\section{Abstrac}

This research is important for the interest that causes the use of new information technologies and communication (NIIC 's) for teaching chemistry in schools, this in order to make the technology on computers, working together with educational research, they can jointly produce material support to facilitate the teaching-learning process of students in the classroom.

\section{Keywords}

Didactic Unit, Troubleshooting, NIIC's, educational software, action research - participatory

\section{Introducción}

Reconociendo que la sociedad ha cambiado y que los procesos de enseñanza/aprendizaje no pueden caracterizarse igual que hace 30 o 40 años, la didáctica de las ciencias junto con otras disciplinas y metadisciplinas se ha interesado por involucrar los avances tecnológicos y de las comunicaciones en el desarrollo de los procesos de aprendizaje.

Partiendo de este hecho, el trabajo que se presenta a continuación cuenta con tres propósitos fundamentales, el primero poder generar una propuesta de enseñanza/aprendizaje de un concepto particular de la química como es el concepto de disoluciones, el aual ha presentado diferentes tipos de dificultades al ser abordado en el aula de dase especialmente por su caracterización matemática, el segundo que esta propuesta este enmarcada en el modelo de resolución de problemas involucrando el contexto cultural, para lo aual se cuenta con el desarrollo de un proyecto 
de aula que reconoce las necesidades y proyecciones del contexto escolar y la aplicación del concepto disoluciones a una actividad dara, cotidiana y tangible y el tercero involucrar el uso de una herramienta tecnológica -software educativo- de tipo simulador para favorecer los proceso prácticos experimentales en el desarrollo de las actividades de dase, este simulador se centrará en el proceso de cultivo hidropónico.

Para poder desarrollar estos propósitos se ha planteado una estructura metodológica que va desde la planeación de las actividades y la estructura del simulador, hasta el seguimiento y recolección de la información, para esto se realiza una investigación de orden cualitativo en donde se analiza la unidad didáctica teniéndola como un estudio de casos y se realiza por medio de Investigación Acción Participativa, utilizando instrumentos de recolección como los diarios de campo (investigadoras y docente), entrevistas semiestructuradas y videos.

\section{Problema}

En la actualidad existen un sin número de investigaciones didácticas que han abordado el problema de la enseñanza y del aprendizaje de determinados conceptos, particularmente en química se puede resaltar el interés centrado y partiaularizado en conceptos estructurantes como Discontinuidad de la materia, cambio químico y cuantificación de relaciones, sin embargo cuando nos centramos en un concepto un poco más espeáfico como es el concepto de disoluciones nos encontramos con diferentes dificultades, como es el tener que establecer un proceso de interacción entre el concepto de discontinuidad de la materia y el de cuantificación de relaciones, también la diferenciación de las disoluciones de las sustancias puras.

Por lo anterior, el problema que orienta esta investigación la siguiente pregunta:

¿Es posible el diseño y la aplicación de una unidad didáctica innovadora, enfocada al concepto de disoluciones, que integre el contexto escolar y las nuevas tecnologías de la información NTIC's, utilizando como trabajo práctico experimental la aplicación de los cultivos hidropónicos, para que motive al estudiante por el aprendizaje de la química?

\section{Objetivo general}

Diseñar y aplicar a partir del modelo de resolución de problemas una unidad didáctica para el concepto de disoluciones que integre el contexto escolar, el trabajo práctico experimental y las nuevas tecnologías de la información NTIC's

\section{Metodología}

La interpretación de la realidad, considera un contexto y un tiempo determinado, sistematizando el estudio a través de descripciones y registros rigurosos de experiencias con el propósito de cambiar, permitiendo establecer propuestas, para la posterior toma de decisiones, innovación y mejora educativa en este contexto.

En este marco, el método de la investigación es Investigación- Acción Participativa y su diseño esta centrado en el Estudio De Casos Múltiples (Comparación Constante). Tanto el método como el diseño de la investigación permitieron describir e interpretar la realidad a través de la intervención a pequeña escala en el funcionamiento del contexto educativo, mediante la aplicación de la unidad didáctica soluciones que cultivan enfocada en el tema de disoluciones, con apoyo de un simulador en cultivos hidropónicos en un grupo experimental y un grupo control. 
Los instrumentos empleados para la recolección de resultados y su posterior análisis se dividen en: resultados obtenidos durante el desarrollo de la unidad didáctica y en los resultados obtenidos a partir del seguimiento continúo del grupo experimental y control.

En los resultados obtenidos durante la aplicación de la unidad didáctica se encuentran los instrumentos de ideas previas, el desarrollo del las $\mathbf{V}$ heuństicas como herramienta reguladora y evaluadora del proceso de enseñanza aprendizaje, el seguimiento continúo del proyecto de dase mediante el desarrollo de los diarios del cultivo hidropónico, del cual se obtienen los resultados propios del proyecto de dase, para finalizar el proceso se realiza la evaluación final, la cual cuenta con tres herramientas para su desarrollo como lo es el desarrollo del diagrama de concepto, el cual busca establecer las relaciones y diferencias que los estudiantes encuentran entre del concepto sugerido disoluciones con el concepto general mezdas. En el diagrama de doble celda el estudiante tiene la posibilidad de establecer la relación entre el concepto de disoluciones y el concepto de cultivos hidropónicos, en la intersección se ubican los conceptos que hacen parte de los dos conceptos asociados, mientras que en los laterales se clasifican los conceptos que solamente se relacionan con uno de los dos conceptos espećficamente. En el desarrollo de la V Heurística, el estudiante tiene la posibilidad de plantear un problema de la vida cotidiana que involucre cada uno de los temas desarrollados durante la aplicación de la unidad didáctica soluciones que cultivan, esto con el fin de determinar la relación que el estudiante establece entre los problemas de la vida cotidiana y la clase de química.

Los resultados sobre el impacto generado en los estudiantes a partir del desarrollo de la unidad didáctica soluciones que cultívan se obtienen mediante el seguimiento del grupo experimental y control con ayuda de las observaciones consignadas en los diarios de campo tanto del profesor como de las investigadoras, en las entrevistas grupales e individuales realizadas a los estudiantes y a un profesor de la institución, quien vivió de cerca el proceso adelantado en la unidad didáctica, la obtención de información mediante los registros fílmicos de la retroalimentación en el uso del simulador y la coevaluación adelantada como parte del proceso de evaluación del estudiante.

\section{Desarrollo de la investigación}

La investigación se desarrolló en el Colegio Oficial Distrital Los Comuneros Oswaldo Guayasamín, de la localidad de Usme con estudiantes del grado once, se trabaja con un grupo experimental y control, con los cuales se realiza la aplicación de la unidad didáctica Soluciones que Cultívan. La unidad didáctica consta de 9 actividades, 1 instrumento de ideas previas, 7 guías y la evaluación final, a partir de la identificación de las ideas previas se organizan las actividades desde la apropiación del concepto de mezdas y de sustancias puras, para ir abordando el tema de disoluciones, para el cual como estructura de aplicación se establece un proyecto de aula orientado a la construcción de un cultivo hidropónico, dentro de las actividades se abordan todos los aspectos de las disoluciones, desde las propiedades físicas, hasta las propiedades químicas. Se hace un énfasis particular en la aplicación del concepto, razón por la cual el trabajo práctico experimentales uno de los ejes vertebradotes de la unidad, así como el uso de un software de tipo simulador en donde se recrea la estructura del cultivo.

Para identificar las diferencias entre los grupos, se desarrolla el trabajo con el simulador de cultivos hidropónicos con el grupo experimental, de esta forma se puede establecer los factores que facilitan el aprendizaje en el estudiante al utilizar herramientas tecnológicas en el aula. 


\section{Análisis de la investigación}

Para el análisis de la información obtenida a lo largo de la aplicación de la unidad didáctica soluciones que Cultivan se toman los resultados obtenidos a partir del desarrollo del trabajo práctico experimental, el instrumento de ideas previas, la evaluación final y las actividades planteadas en el proyecto de clase (Cultivos Hidropónicos), con el fin de establecer los progresos obtenidos por los dos grupos de estudiantes a través de la ejecución de la unidad didáctica. Este análisis tiene como finalidad establecer las diferencias entre el grupo experimental y el grupo control, para de esta forma identificar la incidencia que tienen las TIC's, el trabajo práctico experimental (laboratorios y desarrollo del cultivo hidropónico) en los procesos de enseñanza aprendizaje.

En cuanto al análisis como de la unidad didáctica, se realiza el proceso de triangulación a partir de las categonías de análisis diseñadas durante la fase planificación. Entre los instrumentos empleados para la triangulación se cuentan. Diario de campo de las investigadoras, diario de campo del profesor, entrevistas a estudiantes, entrevista a profesor de la institución y videos de dase.

\section{Conclusiones}

$>$ El desarrollo de la unidad didáctica de disoluciones a partir del modelo de resolución de problemas permite acercar las situaciones cotidianas a los procesos de enseñanza aprendizaje, en donde a partir del análisis de las sustancias que los estudiantes manipulan en su diario vivir logran diferenciar sustancia pura, mezda heterogénea y mezda homogénea, con ello se facilita reconocer las propiedades y características de los sistemas homogéneos, logrando que la matematización de los mismos no sea el eje fundamental del proceso de enseñanza , pero se reconozca como parte esencial al hablar de propiedades como pH y concentración.

> Dadas las características con las que tradicionalmente ha sido abordado el concepto de disoluciones se genera una propuesta de enseñanza - aprendizaje a partir de la resolución de problemas teniendo en cuenta el contexto escolar, lo aual permite buscar en dónde este concepto puede ser aplicado, de tal forma que el desarrollo de aultivos hidropónicos se da como una alternativa para desarrollar este concepto ya que permite su identificación, su caracterización y especialmente la "descomplejización" al ser parte fundamental de un proceso de que soluciona problemas inmediatos -obtención de alimentos- , da aplicación y permite reconocer la importancia de conceptos claves como pH y concentración.

$>$ El desarrollo de actividades de tipo práctico experimental (laboratorio y proyecto de dase), generan en el estudiante un interés particular por el desarrollo de la asignatura ya que se integra el trabajo práctico y la teoría con proyectos de interés para el estudiante como lo es el cultivo hidropónico.

> La aplicación y desarrollo de actividades de tipo virtual permiten generar en el estudiante proceso de apropiación del conocimiento de una forma significativa, lo cual no implica que esta remplace de forma permanente el trabajo práctico experimental real.

$>$ El desarrollo del trabajo práctico experimental de los aultivos hidropónicos facilitó de forma significativa la comprensión por parte de los estudiantes del concepto de disoluciones, ya que trabajaban en tomo a una situación real, en la aual debían tomar decisiones para el óptimo desarrollo y crecimiento del cultivo teniendo en auenta la cantidad y la concentración de la solución nutritiva de las plantas. 
$>$ Se identifica que los estudiantes del grupo experimental (grupo con el aual se desarrolló el proceso de simulación) presentan mayores facilidades en cuanto a la toma de decisiones para establecer las características y condiciones adecuadas para el desarrollo del cultivo hidropónico.

\section{Bibliografía}

Adúriz-Bravo, A., (2001). Integración de la epistemología en la formación del profesorado de ciencias, Tesis doctoral del Programa de Doctorado en Didáctica de las Ciencias Experimentales, Universidad Autónoma de Barcelona.

Adúriz-Bravo, A., (2001). La didáctica de las ciencias como disajplina, Universidad Autónoma de Barcelona

Barbera, O. y Valdes, P. (1996). El trabajo práctico en la enseñanza de las ciencias: una revisión. Enseñanza de las ciencias, 14 (3), 365 - 379.

Begoña, G., (1997). Diseños y programas educativos: pautas pedagógicas para la elaboración de software Barcelona: Ariel Editorial.

Brown, T. L.; Lemay, H. E.; Bursten, B. E. (2004). Química: La ciencia central. México: Prentice - Hall, $9^{a}$ Edición.

Castell, M. (2001). "La era de la información Vol 1,2 y 3 La sociedad red' Madrid, España: Alianza editorial S.A

Cohen, L. y Manion, L, (1990). Métodos de investigación educativa. Editorial La Muralla S.A.

Contreras, L C., (1987). La resolución de problemas ¿UUna panacea metodológica? Enseñanza de las ciencias. Volumen 5. Número 1. Pág. 49-52.

Crespo, E., y Álvarez, T. (2005). Clasificación de las practicas de laboratorio de Física, Universidad de Pinar del Río, Cuba; Revista Pedagogía Universitaria, Vol. 6 (2); Pág. 42 - 49

De Corte, E. (1996). Aprendizaje apoyado en el computador: una perspectiva a partir de investigación acerca del aprendizaje y la instrucción. III Congreso Iberoamericano de Informática Educativa, Nuevas Tecnologías de Información y La Comunicación en la Educación para el Tercer Milenio. Barranquilla (Colombia)

Gallego Badillo, R. y Pérez Miranda, R, (1994) Representaciones y Conceptos Científicos. Un Programa de Investigación, U.P.N., Departamento de Química, Bogotá.

Garća, A, (2005). Aportes del estudio histórico de instrumentos científicos a la formación del profesorado de ciencias; Revista Enseñanza de las Ciencias, Número extra VII Congreso.

García, A., Diazgranados, S., Devia, R., Montoya, A., Sánchez, M., Rivera, C. (2000). Junio, No 2). El cambio didáctico de las concepciones y prácticas docentes en tomo a la resolución de problemas en química: un estudio dirigido al diseño de propuestas de aplicación en el aula. Revista Científica, Centro de Investigaciones y Desarrollo UDFJC, 71 - 113

García, A., Pinilla, J., Y (2007). Pensamiento científico y tecnológica. En Cajiao, F., et al (2007). Colegios públicos de excelencia para Bogotá: Orientaciones para la discusión curricular por campos de conocimiento, propuesta para el cido de educación básica B. Serie documentos de trabajo. Alcaldía Mayor de Bogotá D.C.

García, G. J. J. (2003) Didáctica de las ciencias: resolución de problemas y desarrollo de la creatividad Cooperativa Editorial Magisterio, Bogotá.

García, M. A., Diaz - Granados, S. y Devia, R. Los trabajos prácticos en la enseñanza de las ciencias naturales. Grupo de investigación en didáctica de la química, Universidad Distrital Francisco José de Caldas. En ADÚRZ-BRAVO, A., PREAFÁN, G. A. y BADILLO, E. (2002), Actualización en didáctica de las ciencias y las matemáticas, Cooperativa Editorial Magisterio, Bogotá.

Garrett, R. M (1995) Resolver problemas en la enseñanza de la ciencias. Revista Alambique, didáctica de las ciencias experimentales. Número 5, Pág. 6 - 15.

Kemmis, S. y Mctaggart, R. (1992). Cómo planificar la investigación-acción, Barcelona: Laertes.

Majó, J. y MarquéS, P (2002). "La revolución educativa en la era de la Internet" Barcelona, España: CissPraxis, S.A. 
Padron. A. L. (2005) Las nuevas tecnologías de la información y las comunicaciones (NTIC) en la formación del hombre nuevo. Cuba.

Sanchez. L. A; Cruz. C. S. (2006). Unidad didáctica computacional (UDIC) basada en un simulador para el proceso de destilación simple y fraccionada en el contexto de la enseñanza / aprendizaje de la química. Tesis Universidad Distrital Francisco José de Caldas. Bogotá.

Sanmartí, N. E Izquierdo, M. (2001). Cambio y Conservación en la Enseñanza de las Ciencias ante las TIC Alambique, Didáctica de las Ciencias Experimentales, 29, pp. 71-83.

Sanmartí, N., Gómez, M, (1996). La didáctica de las ciencias: una necesidad, Educación Química, 156 - 168. Marzo.

Whitten, Kenneth. (1999) Química General. Quita edición Mc Graaw Hill. España. 